\title{
Light-Induced Reversible Hierarchical Self-Assembly of Amphiphilic Diblock Copolymers into Microscopic Vesicles with Tunable Optical and Nanocarrier Properties
}

\author{
Yao Sun ${ }^{\ddagger}$ Fei Gao*, Yuan Yao, Haibao Jin, Xinxin Li, Shaoliang Lin* \\ Shanghai Key Laboratory of Advanced Polymeric Materials, Key Laboratory for Ultrafine Materials of Ministry \\ of Education, School of Materials Science and Engineering, East China University of Science and Technology.
}

\section{Supporting Information}

\section{Experimental Section}

\begin{abstract}
Materials.
Monomer 4-vinyl pyridine (4VP) was purchased from Aldrich and purified by distillation prior to polymerization. 2,2'azobis(isobutyronitrile) (AIBN) was used as initiator and recrystallized before use. The reversible additionfragmentation chain transfer (RAFT) agent 4-cyano-4-((phenylcarbonothioyl)thio)pentanoic acid (CPPA) was purchased from Aldrich and used without further purification. Another monomer 6-[4-(4-butylphenylazo) phenoxy] hexyl methacrylate (AzoMA) was synthesized according to our previous work. ${ }^{1}$ Deionized water was collected from Milli-Q system with the resistance of 18.2 M $\Omega$. Other reagents (A.R. grade) were purchased from Adamas-beta and used as received.
\end{abstract}

Synthesis of poly(4-vinylpyridine)-block-poly[6-[4-(4-butyloxyphenylazo) phenoxy] hexyl methacrylate].

Poly(4-vinylpyridine)-block-poly[6-[4-(4-butyloxyphenylazo) phenoxy] hexyl methacrylate] (denoted P4VP-bPAzoMA) diblock copolymer was synthesized by a two-step reversible RAFT polymerization according to our previous work. ${ }^{1}$ Macromolecular RAFT agent P4VP-CPPA was synthesized by a typical RAFT polymerization of 4VP with CPPA initiated by AIBN. Target diblock copolymer P4VP-b-PAzoMA was synthesized via a second RAFT polymerization of AzoMA monomers with P4VP-CPPA. Chemical structure and characterization of as-synthesized diblock copolymer are shown in Figure S1.

Formation of P4VP-b-PAzoMA reverse micelle and crosslinking of P4VP cores.

First, P4VP-b-PAzoMA block copolymers were dissolved in chloroform $\left(\mathrm{CHCl}_{3}\right)$ by stirring at room temperature for $12 \mathrm{~h}$ to obtain a stock solution. To prepare the micelle solution, $3 \mathrm{~mL}$ toluene, a selective solvent for PAzoMA block, 
was added to $3 \mathrm{~mL}$ initial solution with the addition rate of $10 \mathrm{~mL} \mathrm{~h}^{-1}$. After mixing, $\mathrm{CHCl}_{3}$ was completely evaporated at $60{ }^{\circ} \mathrm{C}$ to yield $2 \mathrm{mg} \mathrm{mL}^{-1}$ toluene solution of P4VP- $b$-PAzoMA micelles. Then 1,4-diiodobutane (DIB) was added to the micellar solution to crosslink the $\mathrm{P} 4 \mathrm{VP}$ core at a molar ratio of $\mathrm{I} / 4 \mathrm{VP}=0.5$ by stirring at $45^{\circ} \mathrm{C}$ for 3 days.

\section{Hierarchical self-assembly of P4VP-b-PAzoMA micelles.}

The crosslinked P4VP- $b$-PAzoMA micelles were dispersed in DMF at the concentration of $0.02 \mathrm{mg} \mathrm{mL}^{-1}$ by adding 100 fold DMF to the reverse micelle toluene solution as described above. Subsequently, deionized water was added into the $3 \mathrm{~mL}$ DMF solution at the rate of $0.72 \mathrm{~mL} \mathrm{~h}^{-1}$ under slow stirring. The solution was kept without stirring at room temperature for $48 \mathrm{~h}$. Due to the hydrophobic interaction, the azobenzene blocks tend to aggregate and the subunits progressively self-assembled into compound micelles and vesicles (i.e., hierarchical self-assemblies). For the study of the effect of water content on self-assembly, various amount of water was added into the initial subunit solution.

\section{Light-induced reversible hierarchical self-assembly.}

For the disassembly of vesicles into subunits, the solution was irradiated using a LED lamp with $365 \mathrm{~nm}$ UV light (intensity: $90 \mathrm{~mW} \mathrm{~cm}^{-2}$ ) for $8 \mathrm{~h}$ under stirring. For the re-assembly of subunits into vesicles, the solution was irradiated with $450 \mathrm{~nm}$ visible light for $1 \mathrm{~h}$ and kept in dark for $12 \mathrm{~h}$. All experiments were carried out under ambient condition.

\section{Encapsulation of Nile Red and its light-controlled release.}

$0.04 \mathrm{mg}$ Nile Red was dispersed in $4 \mathrm{~mL}$ of the abovementioned subunits DMF solution. Then, $2 \mathrm{~mL}$ deionized water was dropped into the mixed solution at the rate of $0.72 \mathrm{~mL} \mathrm{~h}^{-1}$ under slow stirring. Accompanied with the hierarchical self-assembly of subunits into vesicles, the Nile Red was introduced into the shell of vesicles due to its hydrophobicity. For the light-controlled release, the Nile Red-loaded vesicles was exposed to $365 \mathrm{~nm}$ UV light irradiation for different times.

\section{Template-grown for Au nanoparticles.}

Precursors $\left(\mathrm{HAuCl}_{4} \cdot 3 \mathrm{H}_{2} \mathrm{O}, 0.5\right.$ molar ratio to $\left.4 \mathrm{VP}\right)$ was directly added into the reverse micelles toluene solution. Due to the strong coordination interaction between metal moieties and pyridinic nitrogen, the precursors were preferentially loaded in the compartment occupied by the P4VP cores within a few hours under mild stirring. Notably, the precursors remained within the P4VP blocks of the subunits during the hierarchical self-assembly of subunits when water was added. For the reduction of metal salts into metal nanoparticles, excess hydrazine hydrate was added as reductant under stirring. 


\section{Characterizations}

Transmission Electron Microscopy (TEM). The morphology of self-assemblies at various stages were measured by Transmission Electron Microscopy (TEM) on JEM-1400 JEOL at an accelerating voltage of $100 \mathrm{kV} .5 \mu \mathrm{l}$ of selfassemblies solution was dropped onto a grid coated with pure carbon film and then dried under room temperature. Some TEM samples were exposed to $\mathrm{I}_{2}$ vapor for staining the P4VP block. The distribution of element composition was mapped by an energy dispersive spectrometer (EDS).

Dynamic Light Scattering (DLS) Measurements. Hydrodynamic radius $R_{h}$ was measured by dynamic light scattering and the laser light scattering (LLS) measurements with a LLS spectrometer (ALV/CGS-5022) equipped with an ALV-High QE APD detector and an ALV-5000 digital correlator using a He-Ne laser (the wavelength $\lambda=632.8 \mathrm{~nm}$ ) as the light source. In DLS measurements, the intensity correlation function was measured at $25^{\circ} \mathrm{C}$ at a scattering angle of $90^{\circ}$ and each sample was measured once for 300 seconds. The obtained intensity autocorrelation function, $G^{(2)}(t)$, is related to the electric field autocorrelation function, $g^{(I)}(t)$, by means of the Siegert relation. $g^{(I)}(t)$ is further related to the characteristic linewidth $(\Gamma)$ distribution $G(\Gamma)$. A line width distribution $G(\Gamma)$ can be calculated by a Laplace inversion of $g^{(I)}(t)$ using the CONTIN program. From the expression $\Gamma=D_{a p p} \cdot q^{2}$, the apparent translational diffusion coefficients $\left(D_{a p p}\right)$ were determined. $\Gamma$ is the decay rate, which is the inverse of the relaxation time $(t) . q$ is the scattering vector defined as $q=(4 \pi n \sin (\theta / 2) / \lambda)$ (where $n$ is the refractive index of the solution, $\theta$ is the scattering angle, and $\lambda$ is the wavelength of the incident laser light in vacuum). The apparent hydrodynamic radius $\left(R_{h}\right)$ can be determined by the Stokes-Einstein relationship $R_{h}=k_{B} T /\left(6 \pi \eta D_{a p p}\right)$, where $k_{B}, T$, and $\eta$ are the Boltzmann constant, the absolute temperature, and the solvent viscosity, respectively.

Ultraviolet-Visible spectrophotometer (UV-vis). The UV-Vis absorbance spectra were obtained using UV-2550 SHIMADZU and the optical density (turbidity) was measured at a wavelength of $690 \mathrm{~nm}$ using a quartz cell.

Fourier Transform Infrared spectroscopy (FT-IR). The Fourier transform infrared spectroscopy measurements were conducted by using Nicolet 5700 FT-IR spectrometer in transmission mode. Fluorescence spectra of the aggregates solution were examined in quartz cell on Fluorolog-3-P.

Confocal Laser Scanning Microscope (CLSM). The suspension of vesicles loaded with Nile Red was imaged with CLSM (Nikon A1R) under a $561 \mathrm{~nm}$ laser illumination. The samples were prepared by placing a drop of suspension on a cover glass and then dried at room temperature.

Gel Permeation Chromatography (GPC). Relative molecular weights and molecular weight distributions were measured by a gel permeation chromatography (GPC) system equipped with a Waters 1515 Isocratic HPLC pump, a Waters 2414 refractive index detector and a set of Waters Styragel columns (HR3 (500-30,000), HR4 (5,000-600,000), and HR5 $(50,000-4,000,000), 7.8 \times 300 \mathrm{~mm}$, particle size $5 \mu \mathrm{m})$. GPC measurements were carried out at $35^{\circ} \mathrm{C}$ using THF

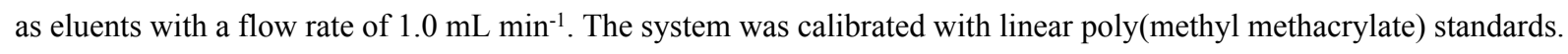




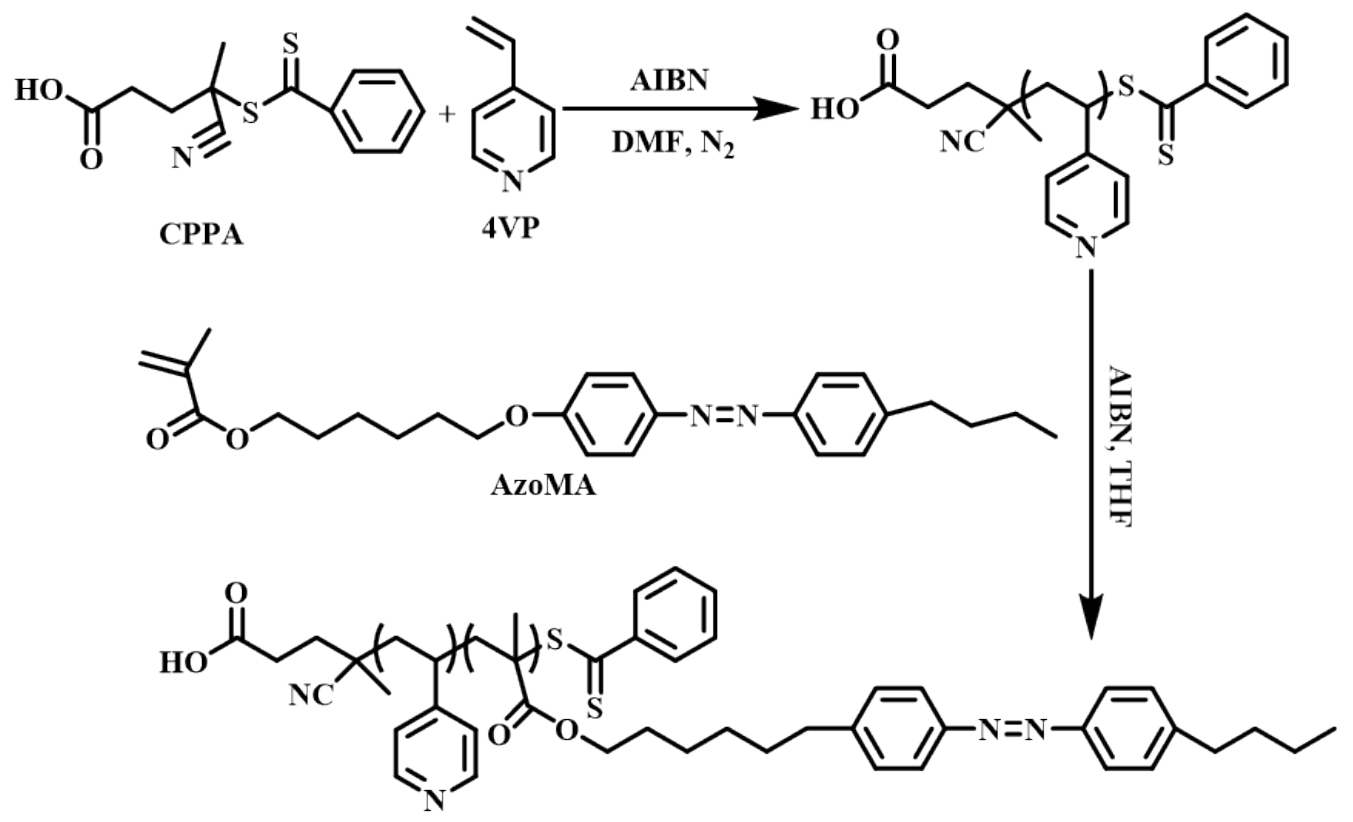

Figure S1. Synthetic route to P4VP-b-PAzoMA block copolymer.
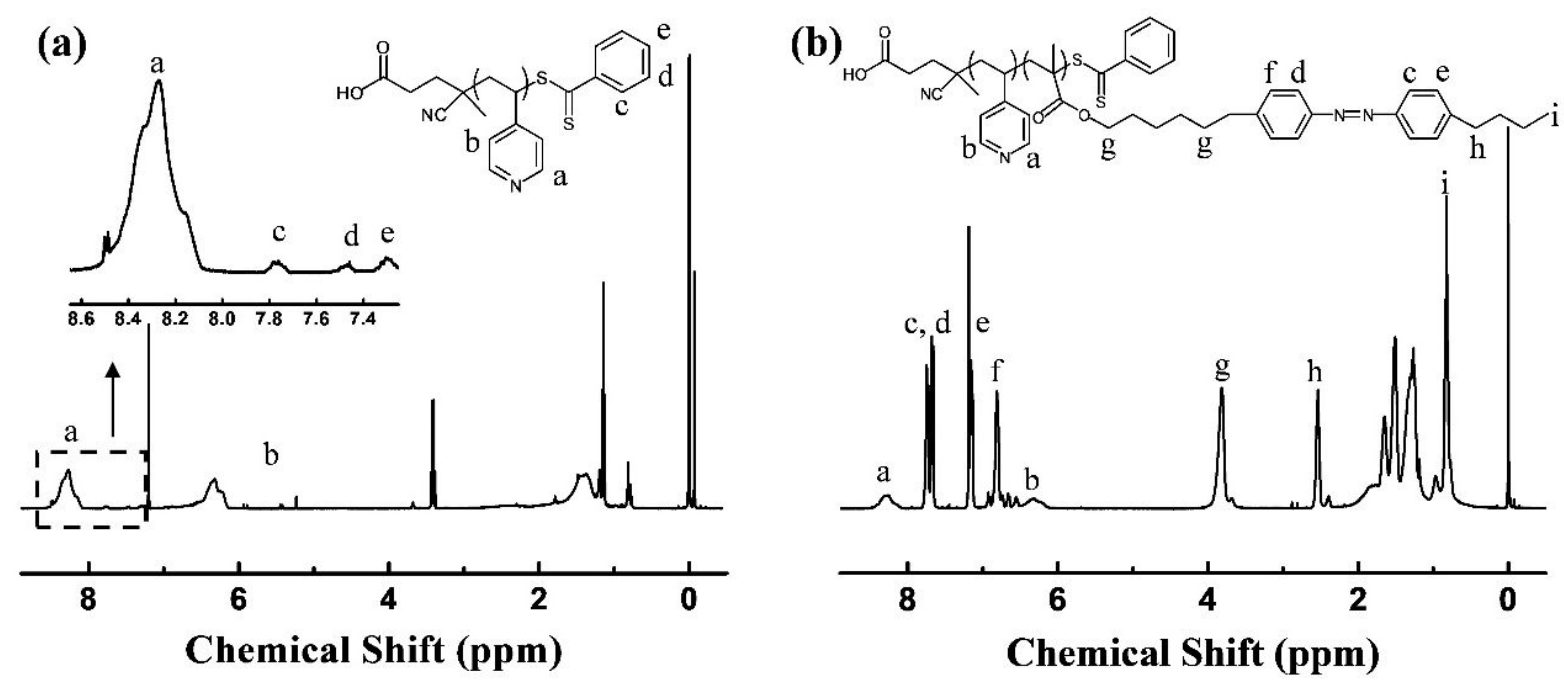

Figure S2. ${ }^{1} \mathrm{H}$ NMR spectra of (a) P4VP-CPPA macro chain transfer agent, and (b) P4VP-b-PAzoMA block copolymer in $\mathrm{CDCl}_{3}$. 


$$
\mathrm{P}_{4 \mathrm{VP}} \text {-b-PAzoMA } 131, M_{n}=2.4 \times 10^{4}, M_{W} / M_{n}=1.27
$$

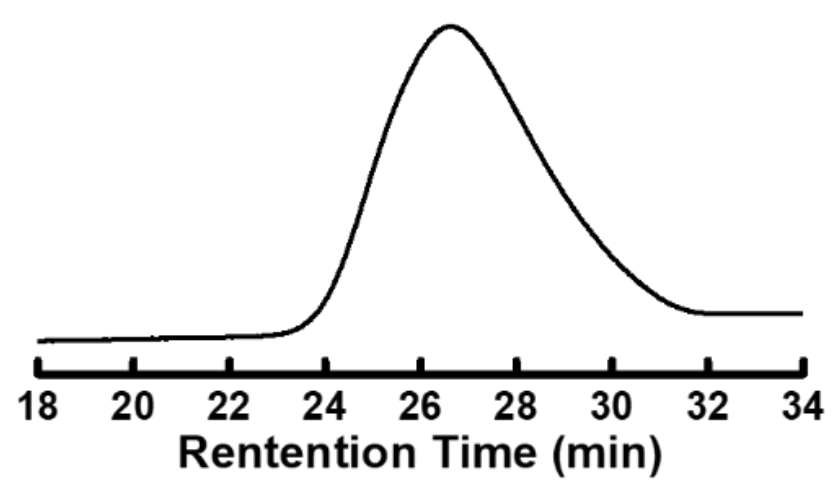

Figure S3. GPC trace of P4VP- $b$-PAzoMA.
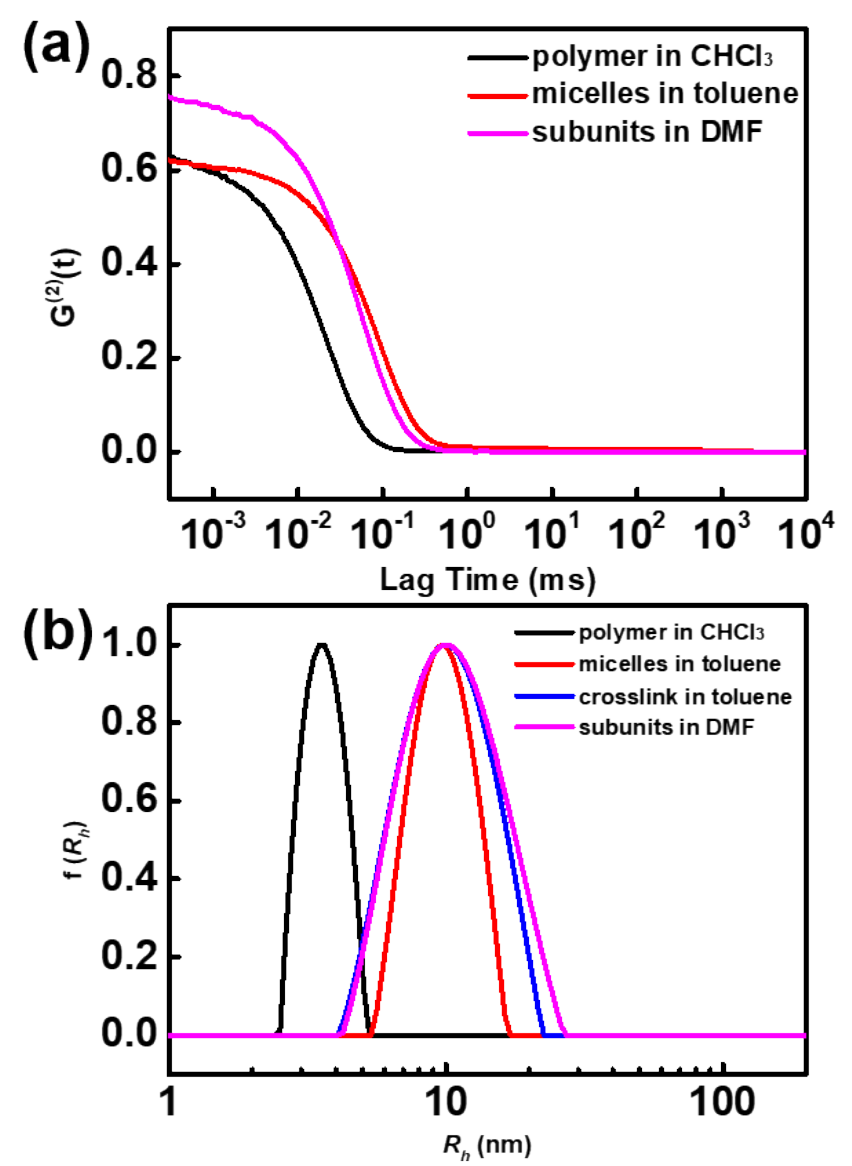

Figure S4. (a) Dynamic light scattering autocorrelation functions and relative fitting, (b) Hydrodynamic radius distributions of P4VP-b-PAzoMA in $\mathrm{CHCl}_{3}$ (black), the initial micelles formed in toluene (red), the crosslinked initial micelles in toluene (blue) and the subunits in DMF (pink). 


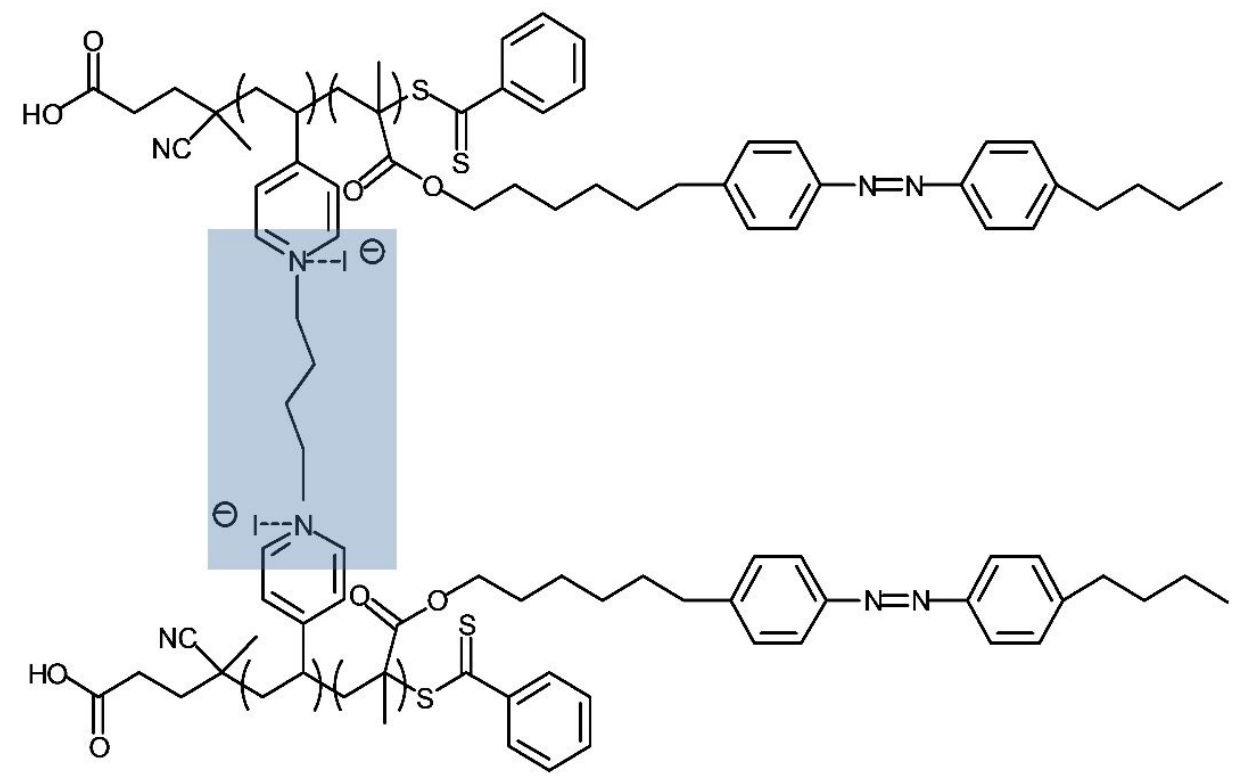

Figure S5. Chemical structures of P4VP-b-PAzoMA diblock copolymers crosslinked by 1,4-diiodobutane (DIB).

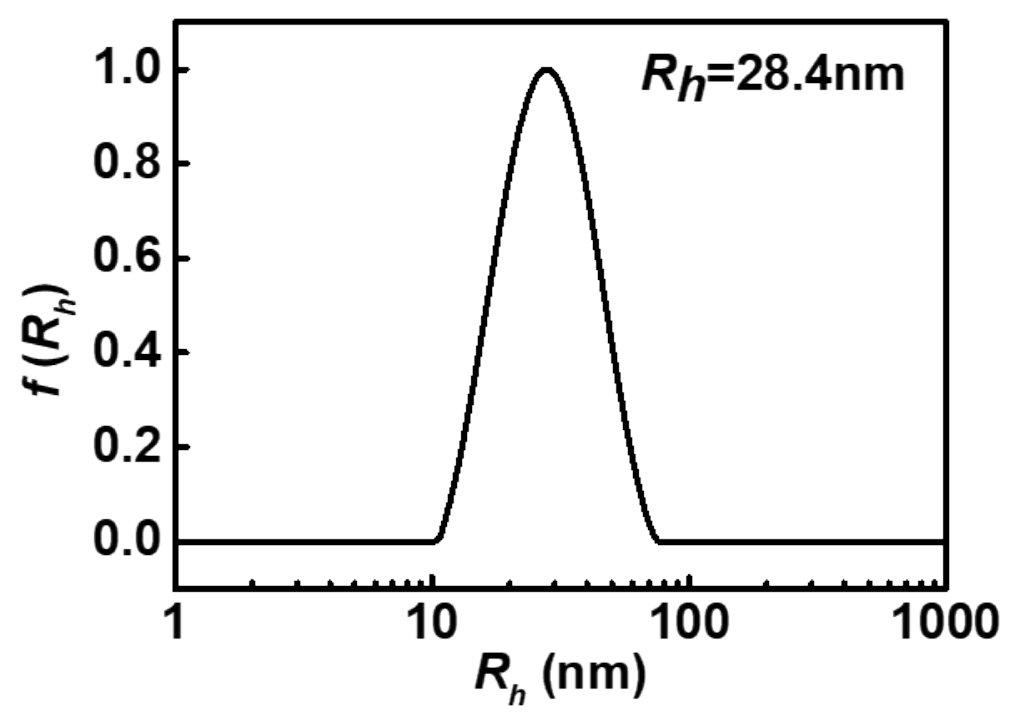

Figure S6. $R_{h}$ values of P4VP- $b$-PAzoMA reverse micelle re-dissolution in $\mathrm{CHCl}_{3}$. 


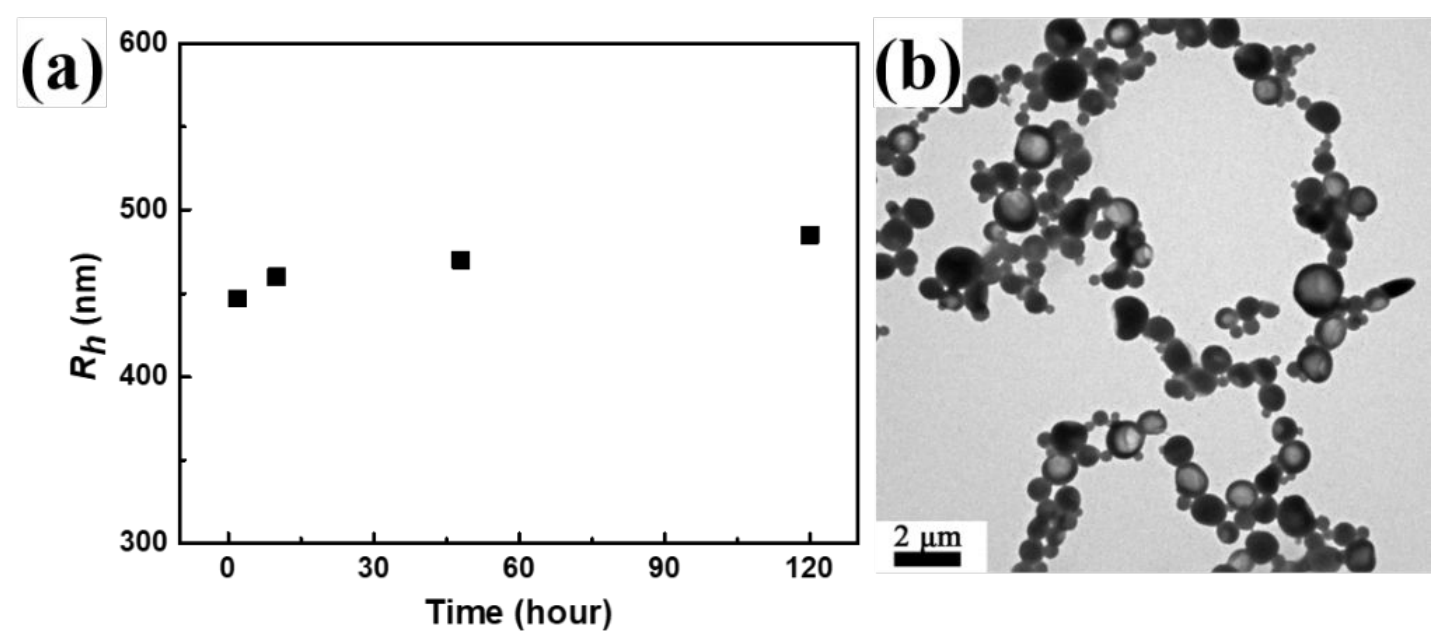

Figure S7. (a) $R_{h}$ of vesicle via by hierarchical self-assembly at different times. (b) TEM images of vesicles remaining stable for 3 months.

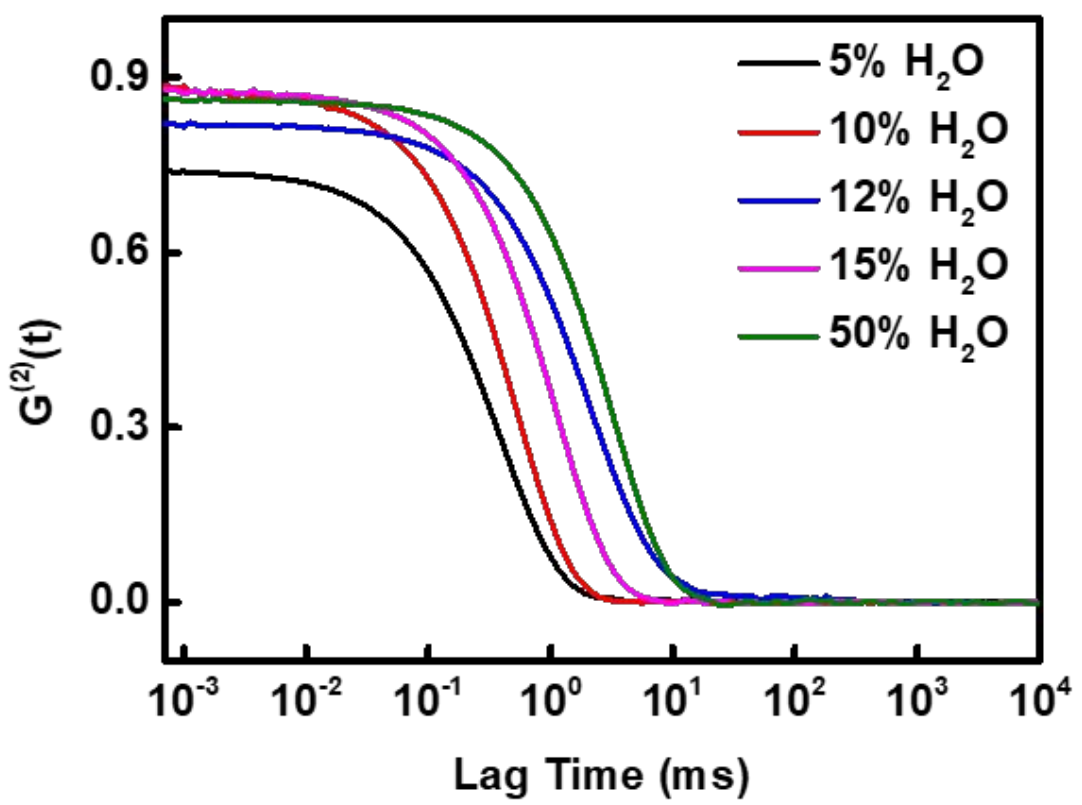

Figure S8. The autocorrelation functions for hydrodynamic radius distributions of hierarchical self-assemblies in DMF with different amounts of water added. 

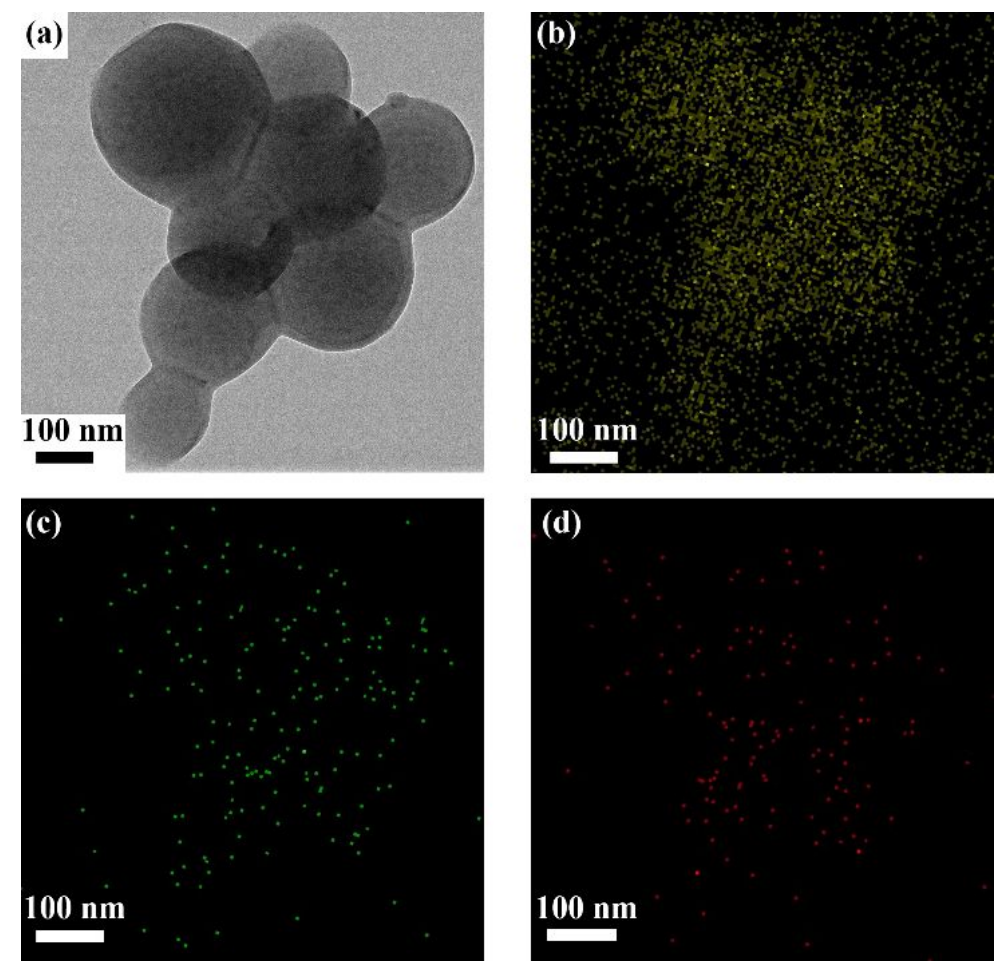

Figure S9. (a) Enlarged TEM image, (b) carbon, (c) nitrogen and (d) iodine element mapping of compound micelles at 10 vol $\%$ water content.
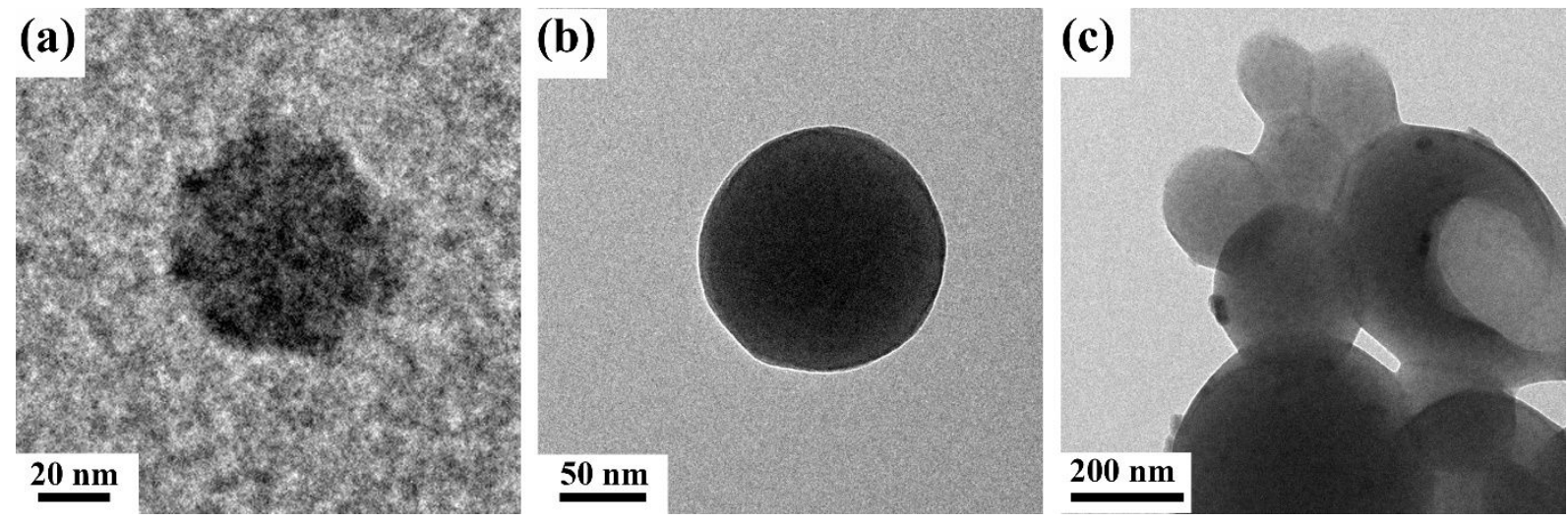

Figure S10. The high magnification TEM images of compound micelles in the mixed $\mathrm{DMF} / \mathrm{H}_{2} \mathrm{O}$ solvents with different water content (a) $5 \mathrm{vol} \%$, (b) $10 \mathrm{vol} \%$ and (c) $12 \mathrm{vol} \%$, respectively. 


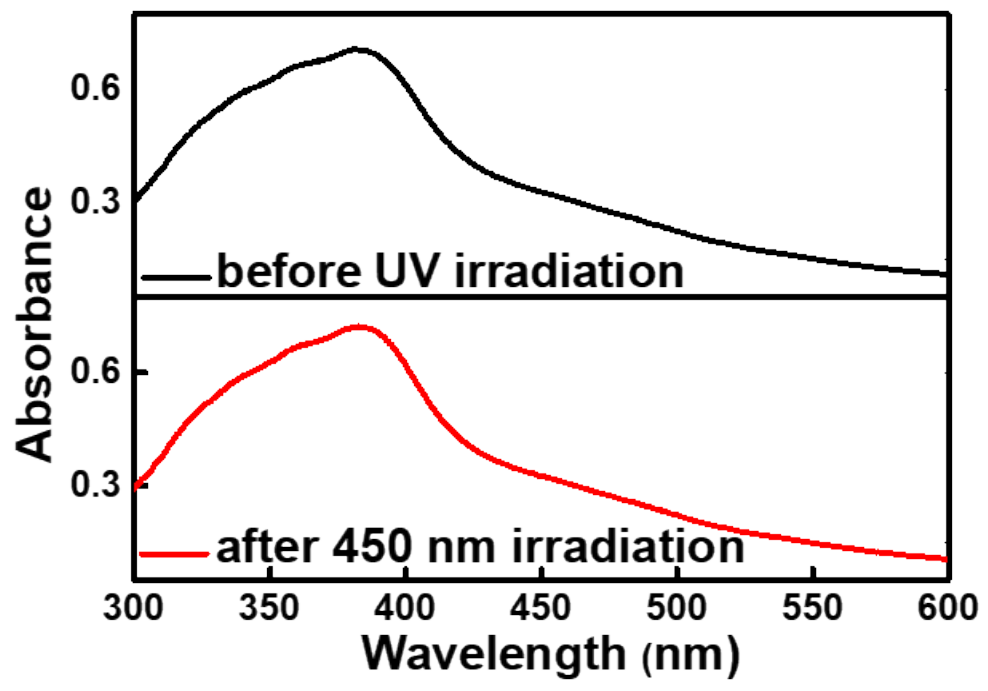

Figure S11. UV-Vis absorption spectra of initial vesicles and recovery vesicles irradiated by $365 \mathrm{~nm} \& 450 \mathrm{~nm}$ light.

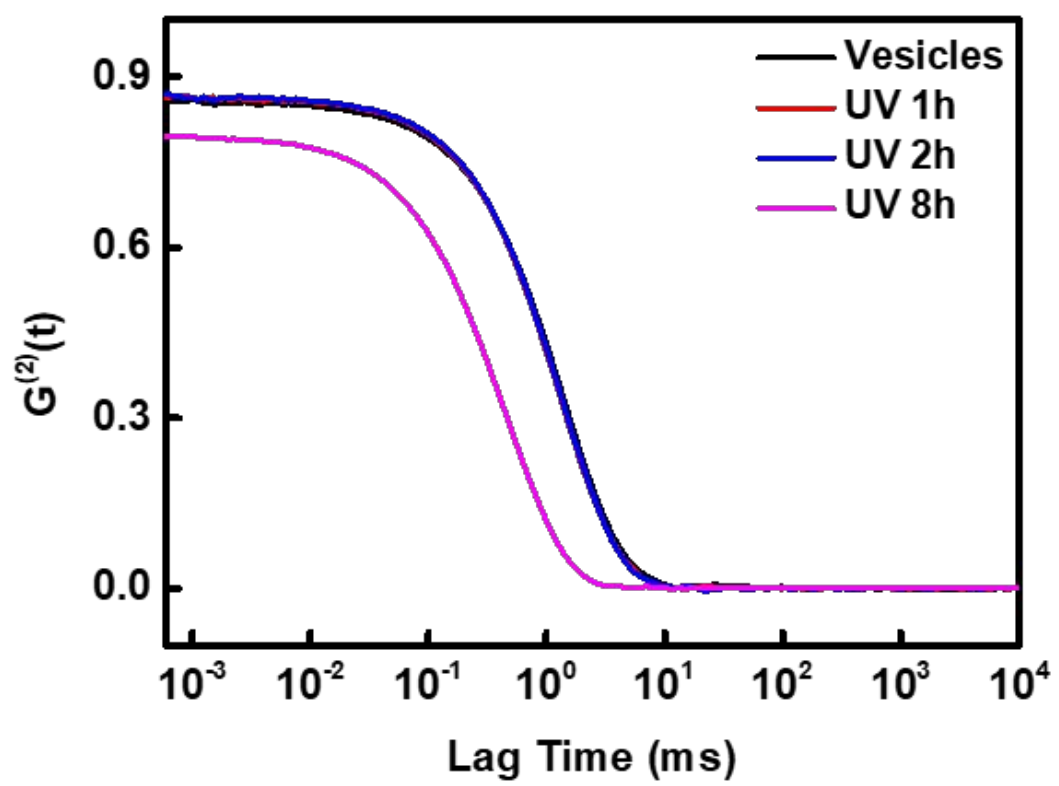

Figure S12. The autocorrelation functions for hydrodynamic radius distributions of vesicles irradiated by UV light for different times. 

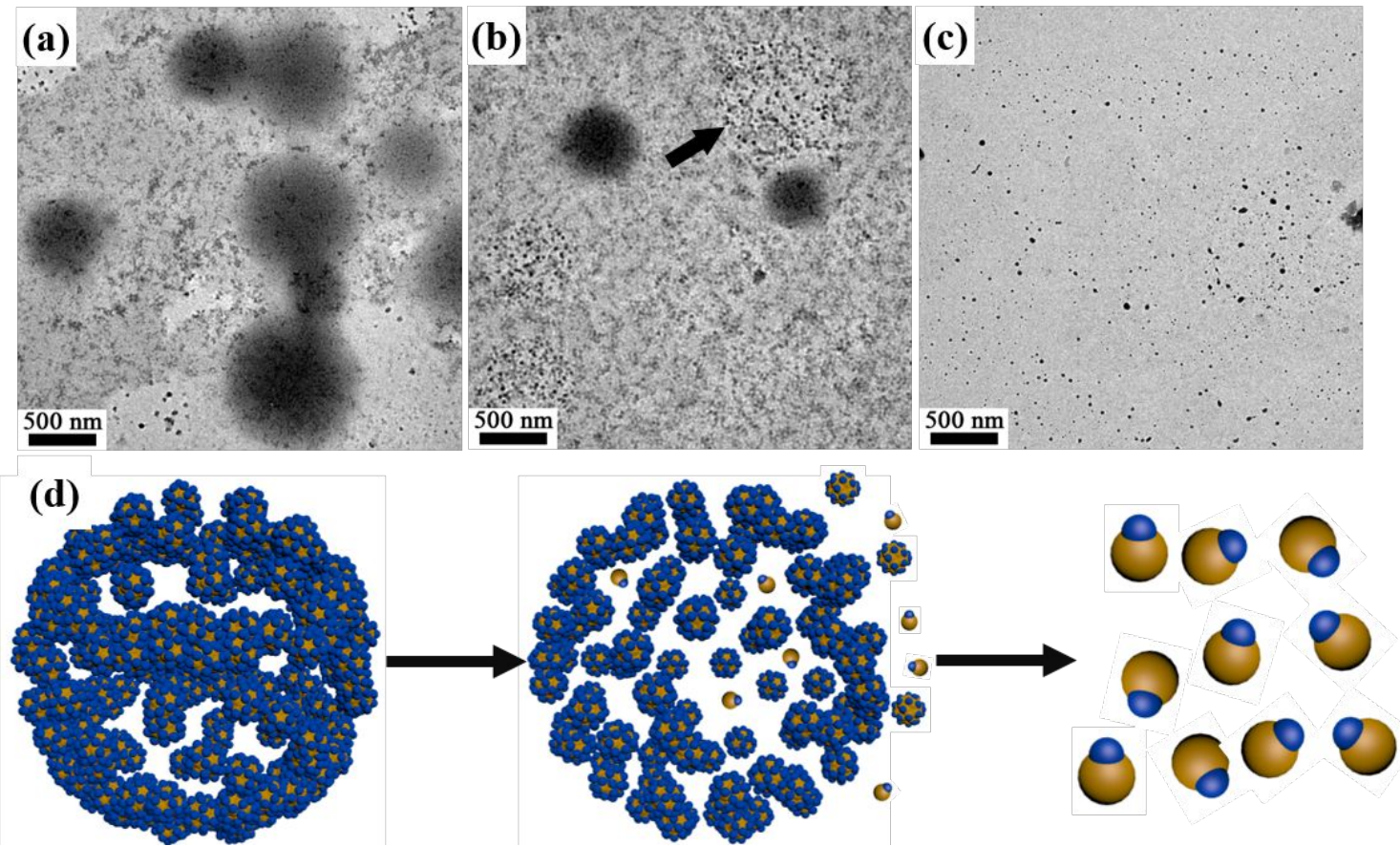

Figure S13. The morphologies of vesicles irradiated by $365 \mathrm{~nm}$ UV light for different times. (a) $1 \mathrm{~h}$, (b) $2 \mathrm{~h}$ and (c) 8 h. (d) Schematic representation of vesicles progressively disrupted into small building blocks (i.e., subunits) via UV irradiation.

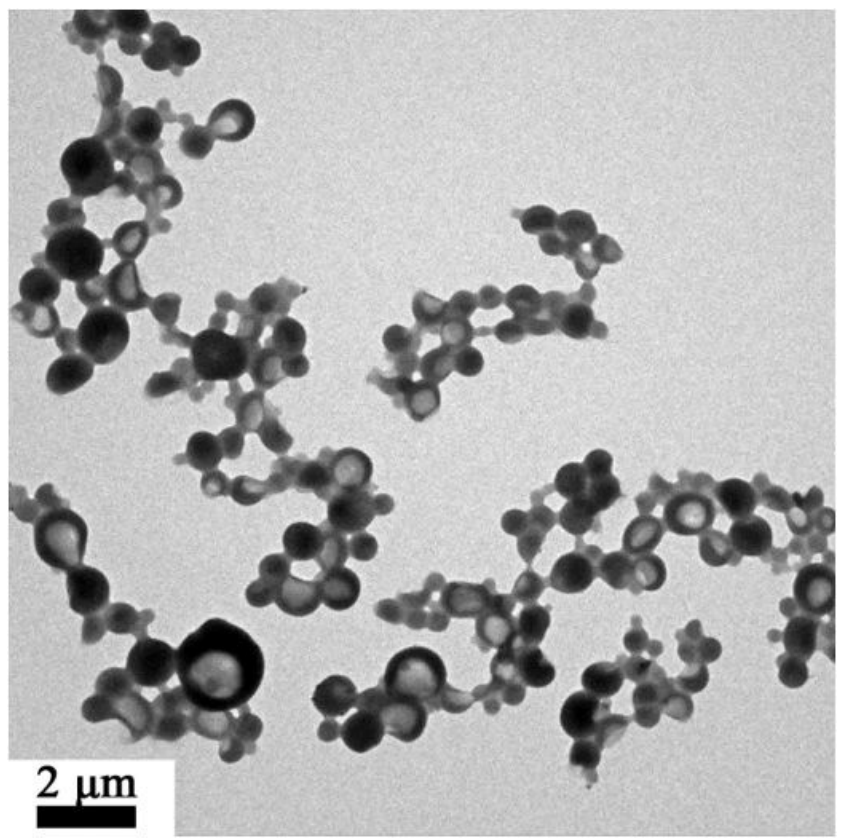

Figure S14. TEM images of hierarchically self-assembled vesicles kept at $\mathrm{T}=60^{\circ} \mathrm{C}$ for $12 \mathrm{~h}$. 


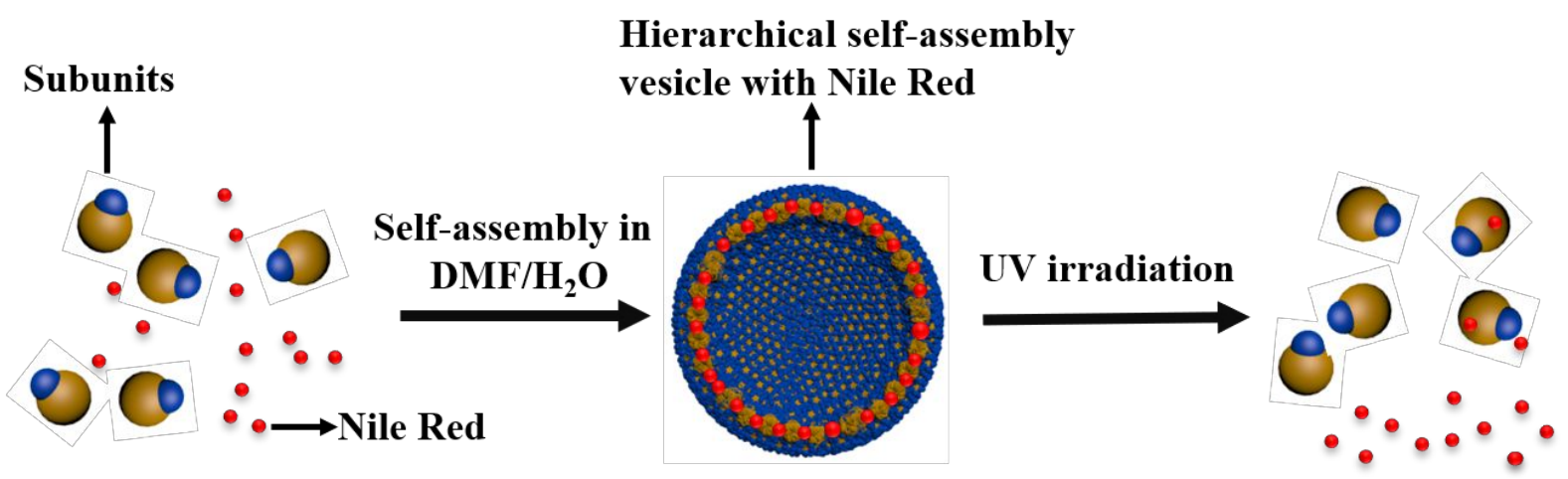

Figure S15. Stepwise representation of Nile Red encapsulation in vesicles via the process of hierarchical self-assembly of P4VP-b-PAzoMA subunits and subsequent controlled release triggered by UV light.
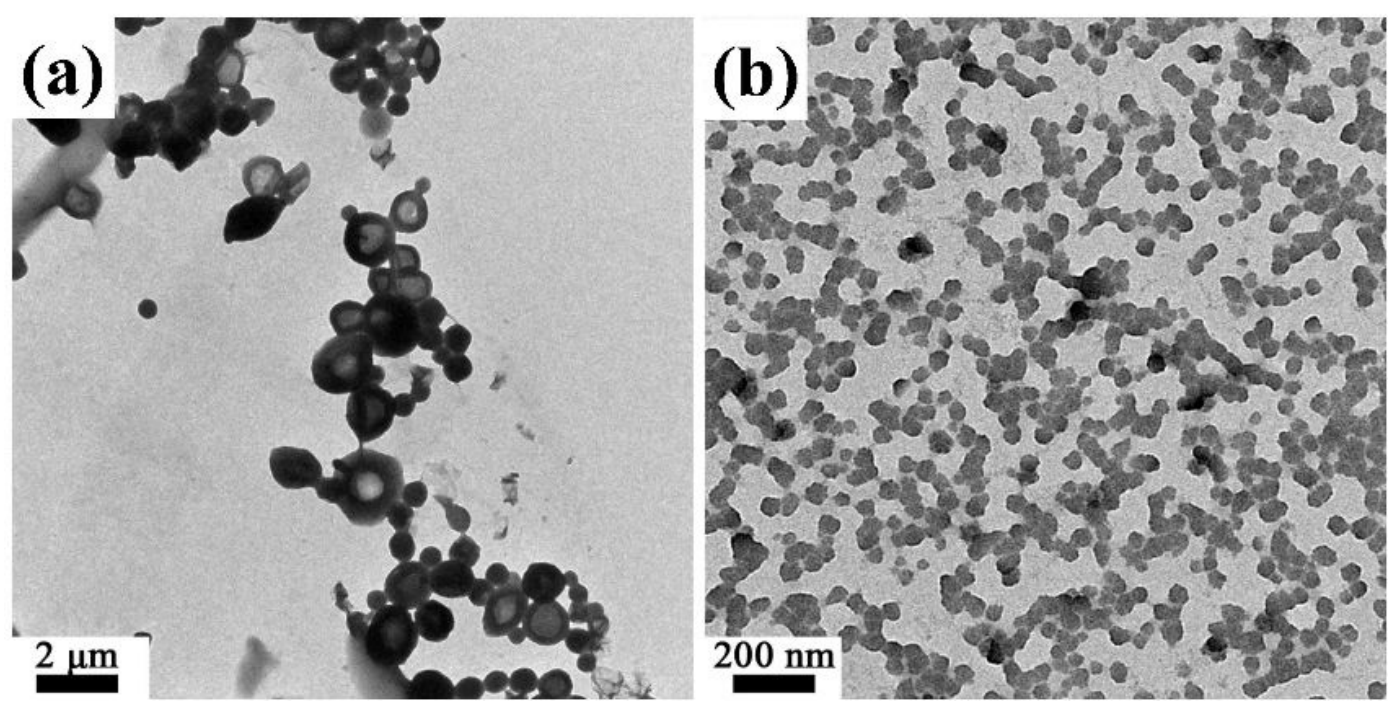

Figure S16. TEM images of the morphology of (a) Nile Red-encapsulated vesicles and (b) disrupted compound micelles coexisted with subunits controlled release by UV light. 

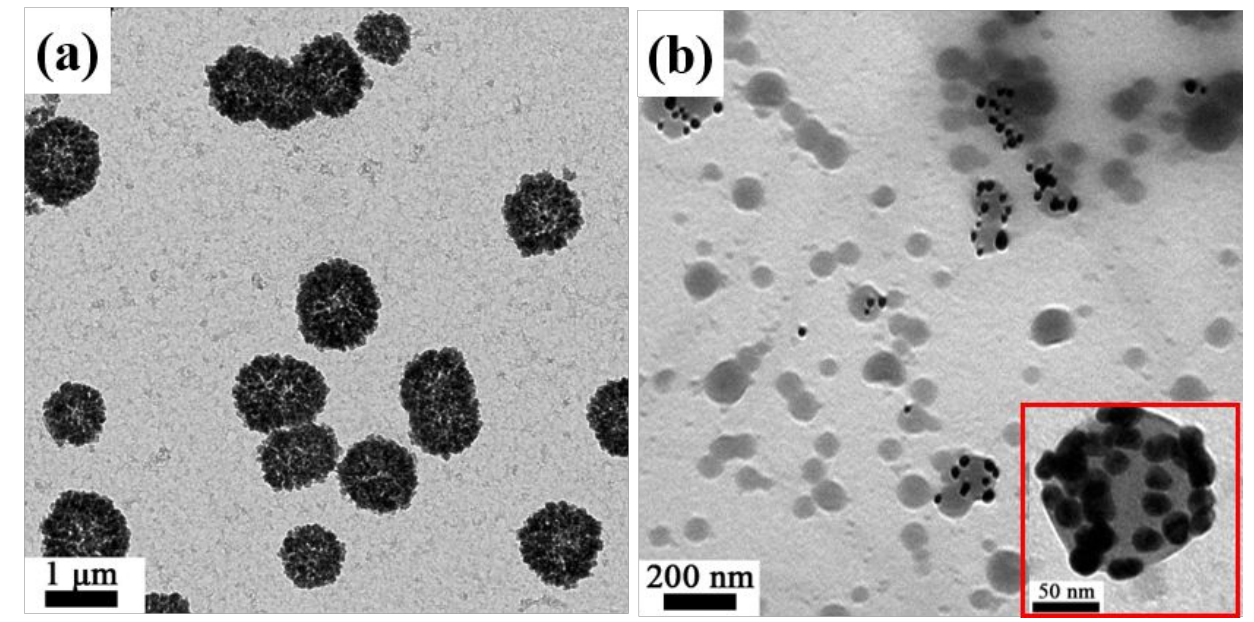

Figure S17. TEM images of (a) Au nanoparticles-containing vesicles and (b) compound micelles and subunits formed after the disruption of vesicles upon UV irradiation and some compound micelles still containing gold nanoparticles due to strong coordination interaction between Au NPs and P4VP blocks.

\section{References}

1. Wang, W.; Du, C.; Wang, X.; He, X.; Lin, J.; Li, L.; Lin, S., Directional Photomanipulation of Breath Figure Arrays. Angew. Chem. Int. Ed. 2014, 53 (45), 12116-12119. 